Physics

Physics Research Publications

\title{
Many-body local fields theory of quasiparticle properties in a three-dimensional electron liquid
}
G. E. Simion
G. F. Giuliani

This paper is posted at Purdue e-Pubs.

http://docs.lib.purdue.edu/physics_articles/754 


\title{
Many-body local fields theory of quasiparticle properties in a three-dimensional electron liquid
}

\author{
George E. Simion* and Gabriele F. Giuliani \\ Department of Physics, Purdue University, West Lafayette, Indiana 47907-2036, USA \\ (Received 29 June 2007; revised manuscript received 16 October 2007; published 24 January 2008)
}

\begin{abstract}
We present a quantitative study of the quasiparticle properties of a three-dimensional electron Fermi liquid. Our approach is based on the theory of the many-body local field factors which we use to include vertex corrections associated with charge and spin fluctuations. Extensive use is made of the results of recent quantum Monte Carlo calculations. Several models for the wave-vector dependence of the spin-antisymmetric manybody local field factor $G_{-}$, for which no numerical results are currently available, are discussed and compared. Both the real and imaginary parts of the self-energy as well as the quasiparticle renormalization constant and the enhancement of the effective mass are calculated in the experimentally accessible range of electron densities. The results obtained by means of the on-shell approximation are critically compared with those given by a self-consistent solution of the Dyson equation. An ultraviolet catastrophe in the Coulomb-hole part of the self-energy is identified and a satisfactory resolution of this impasse is presented. The same many-body local field factors are also used to obtain the Landau interaction function. This allows us to obtain both the spin susceptibility and the proper compressibility of the system.
\end{abstract}

DOI: 10.1103/PhysRevB.77.035131

PACS number(s): 71.10.Ay, 71.18.+y, 71.10.Ca

\section{INTRODUCTION}

Although two-dimensional systems have recently almost supplanted the three-dimensional elemental metals as the system of choice to test microscopic many-body theories of the electron liquid, it is quite useful to further develop theories for the three-dimensional case in view of the fact that a number of methods still see considerable use in the semiquantitative determination of the properties of a large class of three-dimensional metallic and semiconducting systems. $^{1-4}$

In the absence of polarization, the only relevant parameter to the physics of the electron liquid is represented by the dimensionless density parameter $r_{s}$ which, the three dimensions, is given by $r_{s}=\left(\frac{3}{4 \pi n}\right)^{1 / 3} a_{B}^{-1}$, where $n$ is the electron number density and $a_{B}$ the effective Bohr radius. It is widely believed that in the high to metallic density regime, which corresponds to values of $r_{s}$ in the approximate range $0 \leq r_{s}$ $\leq 6$, the electronic system of a three-dimensional metal is in a Fermi liquid state. Since a perturbative treatment of the effects of the Coulomb interaction is significant only for $r_{s} \ll 1$, a number of approximated theoretical schemes have been developed that are designed to cautiously venture into the intermediate coupling regime. Methods range from diagrammatic expansions ${ }^{5}$ to theories based on the many-body local field factor idea. ${ }^{1}$ Quantum Monte Carlo based numerical studies offer an alternative route and have the advantage of a theoretical framework that is accurate and virtually exact for a wide range of densities. ${ }^{6-10}$

Despite intensive efforts, a quantitative characterization of the Fermi liquid regime in the intermediate density range is still to be achieved. In the case of the three-dimensional electron liquid, following a route originally proposed by Hubbard, ${ }^{11}$ vertex corrections were approximately incorporated to this purpose by Rice ${ }^{12}$ through the use of a static many-body local field factor in an attempt to go beyond the random phase approximation (RPA) of Hedin and Lundqvist $^{13}$ and Quinn and Ferrell. ${ }^{14,15}$ This early approxi- mate approach is still quite popular, although it attempts to capture physical effects beyond the RPA by including only the parallel spin many-body local field factor. A number of shortcomings of this theory have been identified and discussed in Refs. 16 and 17.

Following Rice's work, calculations for the threedimensional electron liquid were carried out by Zhu and Overhauser, ${ }^{18}$ who for the first time employed the spinantisymmetric local field factor, and most recently, by means of diagrammatic means, by Rahman and Vignale ${ }^{19}$ and $\mathrm{Ng}$ and Singwi. ${ }^{20,21}$

The many-body local field factor method was more recently rid of serious problems and further developed by Yarlagadda and Giuliani who also provided a modern derivation by making use of the renormalized Hamiltonian approach. ${ }^{16,22}$ A direct application of the technique was provided for the two-dimensional case. ${ }^{23}$ Very recently, a more accurate analysis of the latter was reported in Ref. 24.

While for the two-dimensional electron liquid modern versions of the many-body local field factors were employed in the most recent calculations (see, for instance, Ref. 24), this has never been attempted for the case of the threedimensional electron liquid. To remedy this situation is the purpose of our work.

The recent developments of accurate diffusion quantum Monte Carlo numerical techniques have allowed the precise evaluation of some of the relevant static many-body local field factors. In particular, both the static spin-symmetric ${ }^{8}$ and spin-antisymmetric ${ }^{9}$ many-body local field factors have been obtained for the two-dimensional electron liquid. On the other hand, in view of the magnitude of the numerical effort required, only the corresponding spin-symmetric quantity has been obtained for the three-dimensional case. ${ }^{7}$ Corresponding useful interpolation formulas have been devised as to expedite the use of quantum Monte Carlo results within numerical approaches. ${ }^{25-28}$ The latter have recently found an interesting and elegant application in the virtually exact calculation of the linear charge modulation of the electron density in the presence of an impurity potential (Friedel oscilla- 


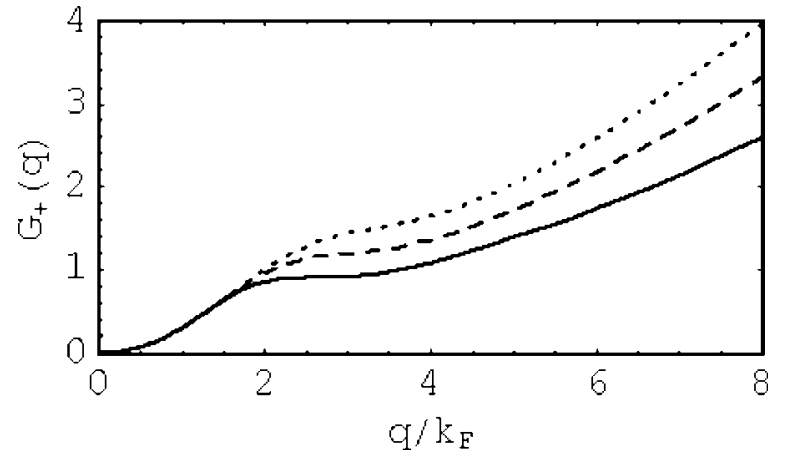

FIG. 1. Wave-vector dependence of the static three-dimensional spin-symmetric many-body local field factor $G_{+}(q, 0)$ as obtained from quantum Monte Carlo simulations via Eq. (31) in the text. Solid line: $r_{s}=1$; dashed line: $r_{s}=2$; dotted line: $r_{s}=4$.

tions), a phenomenon that can be observed and quantified by scanning tunneling microscopy of metallic surfaces. ${ }^{29-31}$ Work on this subject can be found in Refs. 32 and 33 .

The absence of reliable results for the spin-antisymmetric many-body local field factor has necessitated the introduction of several empirical and in general arbitrary models. A large part of our work consists in determining how the physical results of the calculations depend on the specific features of the model used.

We have carried out quantitative calculations of the electronic self-energy, the quasiparticle effective mass, and the renormalization constant, as well as the spin susceptibility enhancement, the effective Landè factor, and the bulk modulus of a three-dimensional electron liquid by including the effects of charge and spin fluctuations by employing increasingly more sophisticated model many-body local field factors.

The paper is organized as follows. Section II develops the general theoretical framework and contains the most important formulas. Section III provides a description of the relevant notions regarding the many-body local field factors as well a series of increasingly complex interpolation formulas. In Sec. IV, these expressions are employed to obtain both the real and the imaginary wave vector and frequency dependent electron self-energy. Section V is devoted to a thorough discussion of the quasiparticle properties including the Coulomb effective mass and the renormalization constant (i.e., the strength of the quasiparticle pole). In Sec. VI, the Landau interaction function is obtained and employed to calculate both the spin enhancement (the static long wavelength limit of the spin susceptibility) and the bulk modulus of the system. Finally, Sec. VII contains our discussion of the results and the conclusions.

\section{GENERAL FORMALISM: THE ELECTRON SELF-ENERGY}

The central object of our study is the wave vector and frequency dependent electron self-energy $\Sigma_{\sigma}(\vec{k}, \omega)$. Once this quantity is known as a function of all its variables, a number of quasiparticle properties can be readily extracted. ${ }^{1}$ For instance, the renormalization constant $Z$, giving the strength of the quasiparticle pole, can be obtained from the frequency dependence as follows:

$$
Z=\frac{1}{1-\left.\frac{1}{\hbar} \frac{\partial \operatorname{Re} \Sigma(k, \omega)}{\partial \omega}\right|_{k=k_{F}, \omega=\epsilon_{F} / \hbar} .}
$$

Another derived important physical property is represented by the quasiparticle energy $E_{Q P}(k)$ which, once the selfenergy is known, can be defined as the self-consistent solution of Dyson's equation,

$$
E_{Q P}(\vec{k})=\varepsilon_{\vec{k}}+\operatorname{Re} \Sigma\left[\vec{k}, E_{Q P}(\vec{k})\right],
$$

where $\varepsilon_{\vec{k}}$ is the unperturbed electron energy. In turn, the effective quasiparticle effective mass can be obtained from the slope of the quasiparticle energy at the Fermi surface,

$$
\frac{1}{m^{*}}=\left.\frac{1}{\hbar^{2} k_{F}} \frac{d E_{Q P}(\vec{k})}{d k}\right|_{k=k_{F}} .
$$

Formally, Eqs. (3) and (2) lead to the expression

$$
\left.\frac{m^{*}}{m}\right|_{D}=\frac{1-\left.\frac{1}{\hbar} \frac{\partial \operatorname{Re} \Sigma(k, \omega)}{\partial \omega}\right|_{k=k_{F}, \omega=\epsilon_{F} / \hbar}}{1+\left.\frac{m}{\hbar^{2} k_{F}} \frac{\partial \operatorname{Re} \Sigma(k, \omega)}{\partial k}\right|_{k=k_{F}, \omega=\epsilon_{F} / \hbar}},
$$

which, besides its formal significance, does also provide a test for the accuracy and consistency of quantitative calculations.

A popular alternative to Eq. (2) is represented by the so-called on-shell approximation (OSA) which consists in taking

$$
E_{O S A}(\vec{k}) \simeq \varepsilon_{\vec{k}}+\operatorname{Re} \Sigma\left(\vec{k}, \varepsilon_{\vec{k}}\right) .
$$

Formally, Eqs. (3) and (5) lead to the approximate expression

$$
\begin{aligned}
& \left.\frac{m^{*}}{m}\right|_{\text {OSA }} \\
& =\left.\frac{1}{1+\frac{1}{\hbar} \frac{\partial \operatorname{Re} \Sigma(k, \omega)}{\partial \omega}+\frac{m}{\hbar^{2} k_{F}} \frac{\partial \operatorname{Re} \Sigma(k, \omega)}{\partial k}}\right|_{k=k_{F}, \omega=\epsilon_{F} / \hbar} .
\end{aligned}
$$

It is well known that the OSA can only be justified in the weak coupling limit. ${ }^{1}$ This caveat must be taken quite seriously since in the case of the two-dimensional electron liquid, for instance, a casual use of the OSA formula leads to a spurious divergence in the quasiparticle effective mass even for experimentally reasonable values of $r_{s}{ }^{24}$ The formal origin of this dangerous pitfall is rather prosaic and can be readily appreciated by comparing the denominator of (approximate) Eq. (6) with that of (exact) Eq. (4). The problem will be discussed below in Sec. V (see Fig. 9).

There are several possible ways of calculating the electron self-energy in an interacting electron liquid. The procedure used in this work is based on the following decomposition: 


$$
\Sigma(\vec{k}, \omega)=\Sigma_{s x}(\vec{k}, \omega)+\Sigma_{c h}(\vec{k}, \omega)
$$

where we have defined the "screened-exchange" part of the self-energy,

$$
\Sigma_{s x}(\vec{k}, \omega)=-\int \frac{d^{3} q}{(2 \pi)^{3}} n_{\vec{k}-\vec{q}}^{0}\left\{v_{\vec{q}}+v_{\vec{q}}^{2}\left[1-G_{+}\left(\vec{q}, \omega-\frac{\epsilon_{\vec{k}-\vec{q}}}{\hbar}\right)\right]^{2} \chi_{n n}\left(\vec{q}, \omega-\frac{\epsilon_{\vec{k}-\vec{q}}}{\hbar}\right)+3 v_{\vec{q}}^{2}\left[G_{-}\left(\vec{q}, \omega-\frac{\epsilon_{\vec{k}-\vec{q}}}{\hbar}\right)\right]^{2} \chi_{S_{z} S_{z}}\left(\vec{q}, \omega-\frac{\epsilon_{\vec{k}-\vec{q}}}{\hbar}\right)\right\},
$$

and the "Coulomb-hole" part,

$$
\Sigma_{c h}(\vec{k}, \omega)=-\frac{\hbar}{\pi} \int \frac{d^{3} q}{(2 \pi)^{3}} \int_{0}^{\infty} d \omega^{\prime} \frac{\operatorname{Im}\left\{v_{\vec{q}}^{2}\left|1-G_{+}\left(\vec{q}, \omega^{\prime}\right)\right|^{2} \chi_{n n}\left(\vec{q}, \omega^{\prime}\right)+3 v_{\vec{q}}^{2}\left|G_{-}\left(\vec{q}, \omega^{\prime}\right)\right|^{2} \chi_{S_{z} S_{z}}\left(\vec{q}, \omega^{\prime}\right)\right\}}{\hbar \omega-\epsilon_{\vec{k}-\vec{q}}-\hbar \omega^{\prime}+i \eta} .
$$

In Eqs. (8) and (9), $\epsilon_{\vec{k}}=\frac{\hbar^{2} k^{2}}{2 m}$ and $v_{\vec{q}}=\frac{4 \pi e^{2}}{q^{2}}$. Moreover, the following relations, appropriate for a homogeneous paramagnetic liquid state, define the charge and spin response functions $\chi_{n n}$ and $\chi_{S_{z} S_{z}}$ and the corresponding spin-symmetric and spin-antisymmetric many-body local field factors $G_{+}(\vec{q}, \omega)$ and $G_{-}(\vec{q}, \omega)$ in terms of the Lindhard response function of noninteracting electrons $\chi_{0}(\vec{q}, \omega)$ as follows:

$$
\chi_{n n}(\vec{q}, \omega)=\frac{\chi_{0}(\vec{q}, \omega)}{1-v_{q}\left[1-G_{+}(\vec{q}, \omega)\right] \chi_{0}(\vec{q}, \omega)}
$$

and

$$
\chi_{S_{z} S_{z}}(\vec{q}, \omega)=\frac{\chi_{0}(\vec{q}, \omega)}{1+v_{q} G_{-}(\vec{q}, \omega) \chi_{0}(\vec{q}, \omega)} .
$$

Within this theoretical framework, the many-body local field factors are introduced to take into account the vertex corrections associated with density spin fluctuations. Their total neglect corresponds to the well-known RPA theory.

The theoretical basis for this approach is well grounded in many-body theory and can, in fact, be justified by following several quite different formal routes. ${ }^{1}$

In particular, a diagrammatic approach for the evaluation of the self-energy has been developed by $\mathrm{Ng}$ and Singwi. ${ }^{20}$ Its starting formula is the exact relationship

$$
\delta \Sigma_{\sigma}(\vec{k}, \omega)=i \hbar \sum_{\sigma^{\prime}} \int \frac{d^{3} \vec{q} d \omega}{(2 \pi)^{4}} I_{\vec{k} \omega \sigma, \vec{q} \omega^{\prime} \sigma^{\prime}}(0) \delta G_{\sigma^{\prime}}\left(\vec{q}, \omega^{\prime}\right),
$$

where $\delta \Sigma_{\sigma}(\vec{k}, \omega)$ and $\delta G_{\sigma}(\vec{q}, \omega)$ are corresponding changes in the self-energy and the Green's function, while $I_{\vec{k} \omega \sigma, \vec{q} \omega^{\prime} \sigma^{\prime}}(0)$ is the irreducible particle-hole interaction with zero momentum and energy transfer. Progress is made by making use of the following "local approximation" originally proposed by Vignale and Singwi: ${ }^{34,35}$

$$
I_{\vec{k} \omega \sigma, \vec{q} \omega^{\prime} \sigma^{\prime}}(0) \simeq V_{\sigma \sigma^{\prime}}^{e f f}\left(\vec{k}-\vec{q}, \omega-\omega^{\prime}\right),
$$

which amounts to express the irreducible particle-hole interaction in terms of an effective local interaction $V_{\sigma \sigma^{\prime}}^{e f f}(\vec{k}, \omega)$.
At this point, the self-energy can be approximately determined only up to an integration constant (this observation is far from trivial and will acquire further relevance later in our discussion) from

$$
\Sigma_{\sigma}(\vec{k}, \omega)=i \hbar \sum_{\sigma^{\prime}} \int \frac{d^{3} \vec{q} d \omega}{(2 \pi)^{4}} V_{\sigma \sigma^{\prime}}^{e f f}\left(\vec{k}-\vec{q}, \omega-\omega^{\prime}\right) G_{\sigma^{\prime}}\left(\vec{q}, \omega^{\prime}\right) .
$$

A corresponding diagrammatic analysis leads to what is referred to as the Kukkonen-Overhauser expression for the effective interaction $V_{\sigma \sigma^{\prime}}^{e f f},{ }^{36-38}$

$$
\begin{aligned}
V_{\sigma \sigma^{\prime}}^{e f f}(\vec{q}, \omega)= & \left\{v_{\vec{q}}+v_{\vec{q}}^{2}\left[1-G_{+}(\vec{q}, \omega)\right]^{2} \chi_{n n}(\vec{q}, \omega)\right. \\
& \left.+v_{\vec{q}}^{2}\left[G_{-}(\vec{q}, \omega)\right]^{2} \chi_{S_{z} S_{z}}(\vec{q}, \omega)\right\} \delta_{\sigma \sigma^{\prime}} \\
& +2 v_{\vec{q}}^{2}\left[G_{-}(\vec{q}, \omega)\right]^{2} \chi_{S_{z} S_{z}}(\vec{q}, \omega) \delta_{\sigma-\sigma^{\prime}},
\end{aligned}
$$

where the response functions $\chi_{n n}$ and $\chi_{S_{z} S_{z}}$ are defined in terms of local many-body field factors $G_{+}(\stackrel{\vec{q}}{q}, \omega)$ and $G_{-}(\vec{q}, \omega)$ as done above. At this point, Eqs. (8) and (9) are readily obtained by inserting Eq. (15) into Eq. (14).

Further insight into the significance and the origin of Eqs. (7)-(11) can be obtained by arriving at an equivalent result by means of an alternative approach based on the renormalized Hamiltonian method. This procedure was pioneered by Hamann and Overhauser and later fully exploited by Yarlagadda and Giuliani to develop a renormalization based picture of the Fermi liquid. ${ }^{1,16,37,38}$ The approach is based on the distinction between "test electrons" (which will ultimately become the quasiparticles) and the remaining electronic degrees of freedom that are treated as a screening medium capable of both charge and spin fluctuations. ${ }^{39}$

From a formal point of view, the fully interacting Hamiltonian describing the whole set of degrees of freedom of the original system is at first separated into a test-electron part, a dielectric medium part, and a term coupling the test electrons with the medium,

$$
\hat{H}_{E G}=\hat{H}_{e}+\hat{H}_{m}+\hat{H}_{\text {int }} .
$$

The test-electron Hamiltonian $\hat{H}_{e}$ is, in particular, expressed as 


$$
\begin{aligned}
\hat{H}_{e}= & \sum_{\vec{q}, \sigma} \epsilon_{\vec{q}} \hat{c}_{\vec{q}, \sigma} \hat{c}_{\vec{q}, \sigma}^{\dagger} \\
& +\frac{1}{2 V_{\vec{p}_{1}, \vec{p}_{2}, \vec{q}}} \sum_{\sigma_{1}, \sigma_{2}} v_{\vec{q}} \hat{c}_{\vec{p}_{1}+\vec{q}, \sigma_{1}}^{\dagger} \hat{c}_{\vec{p}_{2}-\vec{q}, \sigma_{2}}^{\dagger} \hat{c}_{\vec{p}_{2}, \sigma_{2}} \hat{c}_{\vec{p}_{1}, \sigma_{1}},
\end{aligned}
$$

where $\hat{c}_{\vec{p}, \sigma}^{\dagger}$ and $\hat{c}_{\vec{p}, \sigma}$ are the corresponding creation and annihilation operators. $\hat{H}_{m}$ has a similar expression in terms of the operators $\hat{b}_{\vec{p}, \sigma}^{\dagger}$ and $\hat{b}_{\vec{p}, \sigma}$ creating and destroying electrons of the medium.

The last term $\hat{H}_{\text {int }}$ represents the interaction between the electrons and the dielectric medium. Keeping only the terms that separately conserve the number of test electrons and that of medium electrons, we write

$$
\begin{aligned}
\hat{H}_{i n t} \simeq & \frac{1}{2 V} \sum_{\vec{p}_{1}, \vec{p}_{2}, \vec{q}} \sum_{\sigma_{1}, \sigma_{2}} v_{\vec{q}} \hat{c}_{\vec{p}_{1}+\vec{q}, \sigma_{1}}^{\dagger} \hat{b}_{\vec{p}_{2}-\vec{q}, \sigma_{2}}^{\dagger} \hat{b}_{\vec{p}_{2}, \sigma_{2}} \hat{c}_{\vec{p}_{1}, \sigma_{1}} \\
& +\frac{1}{2 V} \sum_{\vec{p}_{1}, \vec{p}_{2}, \vec{q}} \sum_{\sigma_{1}, \sigma_{2}} v_{\vec{q}} \hat{c}_{\vec{p}_{1}+\vec{q}, \sigma_{1}}^{\dagger} \hat{b}_{\vec{p}_{2}-\vec{q}, \sigma_{2}}^{\dagger} \hat{b}_{\vec{p}_{2}, \sigma_{1}} \hat{c}_{\vec{p}_{1}, \sigma_{2}} .
\end{aligned}
$$

This expression can be rewritten in terms of the density and spin fluctuations of the medium. Defining $\Delta_{\vec{p}}(\vec{q})=\epsilon_{\vec{p}}$ $-\epsilon_{\vec{p}-\vec{q}}$, we have

$$
\begin{aligned}
\hat{H}_{i n t} \simeq & \frac{1}{V} \sum_{\vec{p}, \vec{q}, \sigma} v_{\vec{q}}\left\{1-G_{+}\left[\vec{q}, \Delta_{\vec{p}}(\vec{q})\right]\right\} \hat{n}_{-\vec{q}} \hat{c}_{\vec{p}-\vec{q}, \sigma_{1}}^{\dagger} \hat{c}_{\vec{p}, \sigma_{1}} \\
& -\frac{1}{V_{\vec{q}, \vec{k}, \sigma_{1}, \sigma_{2}}} v_{\vec{q}} G_{-}\left[\vec{q}, \Delta_{\vec{p}}(\vec{q})\right] \hat{\vec{S}}_{-\vec{q}} \cdot\left(\hat{c}_{\vec{p}-\vec{q}, \sigma_{1}}^{\dagger}[\boldsymbol{\sigma}]_{\sigma_{1} \sigma_{2}} \hat{c}_{\vec{p}, \sigma_{2}}\right),
\end{aligned}
$$

where $\hat{n}_{\vec{q}}=\sum_{\vec{p}, \sigma} \hat{b}_{\vec{p}-\vec{q}, \sigma}^{\dagger} \hat{b}_{\vec{p}, \sigma}$ and $\hat{\vec{S}}_{\vec{q}}=\sum_{\vec{p}, \sigma, \sigma^{\prime}} \hat{b}_{\vec{p}-\vec{q}, \sigma}^{\dagger}[\boldsymbol{\sigma}]_{\sigma \sigma^{\prime}} \hat{b}_{\vec{p}, \sigma^{\prime}}$ are, respectively, the density and spin-density operators for the dielectric medium. The many-body local field factors $G_{ \pm}$ strategically appear here in order to account for the fact that the interaction between test electrons and medium charge and spin fluctuations is an effective one. This ultimately introduces exchange and correlation effects in a self-consistent way.
The procedure continues with the elimination of the interaction between the test electrons and the medium via a canonical transformation with generator $\hat{T}$,

$$
\hat{H}^{\prime}=\exp (i \hat{T}) \hat{H}_{E G} \exp (-i \hat{T}) .
$$

$\hat{T}$ is chosen in such a way as to eliminate the interaction to first order, i.e.,

$$
\left[\hat{T}, \hat{H}_{e}+\hat{H}_{m}\right]=i \hat{H}_{i n t} .
$$

The resulting Hamiltonian is then averaged over the ground state $\mid 0<$ of the medium in order to arrive to an effective Hamiltonian containing only the test electron degrees of freedom. This gives

$$
\hat{H}_{Q P} \equiv\left\langle 0\left|\hat{H}^{\prime}\right| 0\right\rangle=\hat{H}_{e}+\frac{i}{2}\left\langle 0\left|\left[\hat{T}, \hat{H}_{\text {int }}\right]\right| 0\right\rangle,
$$

an expression that contains suitable renormalized quadratic and quartic forms in the test-electron creation and destruction operators, the latter playing the role of an effective quasiparticle-quasiparticle interaction. At this point, the quasiparticle energies can be calculated by means of a simple perturbative calculation to first order in such an effective interaction. After some standard manipulations, one arrives to the sought expression for the quasiparticle energy,

$$
E_{Q P}(\vec{k})=\epsilon_{\vec{k}}+E_{s x}(\vec{k})+E_{c h}(\vec{k}) .
$$

In Eq. (23), the screened-exchange term $E_{s x}(\vec{k})$ is given by

$$
\begin{aligned}
E_{s x}(\vec{k})= & -\int \frac{d^{3} q}{(2 \pi)^{3}} n_{\vec{k}-\vec{q}}^{0}\left\{v_{\vec{q}}+v_{\vec{q}}^{2}\right. \\
& \times\left|1-G_{+}\left(\vec{q}, \frac{\epsilon_{\vec{k}}-\epsilon_{\vec{k}-\vec{q}}}{\hbar}\right)\right|^{2} \chi_{n n}\left(\vec{q}, \frac{\epsilon_{\vec{k}}-\epsilon_{\vec{k}-\vec{q}}}{\hbar}\right) \\
& \left.+3 v_{\vec{q}}^{2}\left|G_{-}\left(\vec{q}, \frac{\epsilon_{\vec{k}}-\epsilon_{\vec{k}-\vec{q}}}{\hbar}\right)\right|^{2} \chi_{S_{z} S_{z}}\left(\vec{q}, \frac{\epsilon_{\vec{k}}-\epsilon_{\vec{k}-\vec{q}}}{\hbar}\right)\right\},
\end{aligned}
$$

while the corresponding Coulomb-hole part $E_{c h}(\vec{k})$ reads

$$
E_{c h}(\vec{k})=-\frac{\hbar}{\pi} \int \frac{d^{3} q}{(2 \pi)^{3}} \int_{0}^{\infty} d \omega^{\prime} \frac{\operatorname{Im}\left\{v_{\vec{q}}^{2}\left|1-G_{+}\left(\vec{q}, \omega^{\prime}\right)\right|^{2} \chi_{n n}\left(\vec{q}, \omega^{\prime}\right)+3 v_{\vec{q}}^{2}\left|G_{-}\left(\vec{q}, \omega^{\prime}\right)\right|^{2} \chi_{S_{z} S_{z}}\left(\vec{q}, \omega^{\prime}\right)\right\}}{\epsilon_{\vec{k}}-\epsilon_{\vec{k}-\vec{q}}-\hbar \omega^{\prime}+i \eta} .
$$

Notice that the expression of Eq. (23) for the quasiparticle energy can also be obtained by making use of the OSA in Eq. (7).

The decomposition into a screened-exchange part and a Coulomb-hole part is of course not unique. An alternative representation of the self-energy can be obtained in terms of what are commonly referred to as line and residue components. The existence of equivalent expressions for calculating the self-energy allows us to check the accuracy and validity of our calculations. The connection with the formulas derived here is discussed in the Appendix.

\section{GENERAL FORMALISM: MANY-BODY LOCAL FIELD FACTORS}

The many-body local field factors $G_{+}$and $G_{-}$represent the key ingredients for the evaluation of the electron self- 
energy from the previous section. Although some of their properties are known, there is no knowledge of the full wave-vector and frequency dependence of these important quantities. Most of the known facts known about $G_{+}$and $G_{-}$ are discussed in Ref. 1

\section{A. Early interpolation formulas}

Following a long tradition that dates back to Hubbard, ${ }^{11}$ the first step in the handling of the formulas for the quasiparticle energy and the electron self-energy is to approximate the many-body local fields with their static limits $G_{ \pm}(q)$ $=G_{ \pm}(\vec{q}, \omega)$. Although this step greatly simplifies the problem, the next difficulty consists in the fact that not even these functions of the wave vector are well known. Accordingly, a number of more or less successful interpolation formulas have been devised in order to approximately deal with the problem.

Generalizing Hubbard's original expression, Iwamoto and Pines ${ }^{40}$ have proposed an interpolation formula designed to correctly reproduce the long wavelength limit of $G_{ \pm}(q)$,

$$
G_{ \pm}(q)=\frac{1}{2}\left(\frac{q^{2}}{q^{2}+q_{\uparrow \uparrow}^{2}} \pm \frac{q^{2}}{q^{2}+q_{\uparrow \downarrow}^{2}}\right),
$$

where the parameters $q_{\uparrow \uparrow}$ and $q_{\uparrow \downarrow}$ are then chosen to fit the compressibility and the spin susceptibility results obtained through independent methods. Iwamoto and Pines used, for instance, the results obtained by Geldart and Vosko ${ }^{41}$ using the Monte Carlo method.

In particular, the long wavelength limit of the symmetric many-body local field $G_{+}(q)$ is related to the many-body enhancement of the compressibility via ${ }^{1,7}$

$$
\lim _{q \rightarrow 0} \frac{G_{+}(q, 0)}{q^{2}}=\frac{1}{\kappa_{3}^{2}}\left(1-\frac{K_{0}}{K}\right)=\frac{1}{4 k_{F}^{2}}-\frac{1}{4 \pi e^{2}} \frac{d \mu_{c}}{d n},
$$

where $K$ and $K_{0}$ are the compressibilities for the interacting and noninteracting electron liquid, $\mu_{c}$ is the correlation contribution to the chemical potential, and $\kappa_{3}=4 \pi e^{2} N(0)$ is the Thomas-Fermi vector in three dimensions.

The same limit for the antisymmetric many-body local field $G_{-}(q)$ is related to the many-body enhancement of the spin susceptibility via

$$
\lim _{q \rightarrow 0} \frac{G_{-}(q, 0)}{q^{2}}=\frac{1}{\kappa_{3}^{2}}\left(1+\frac{1}{\mu_{B}^{2}} \frac{\chi_{P}}{\chi_{S_{z} S_{z}}}\right),
$$

with $\chi_{P}$ being the Pauli susceptibility of a noninteracting electron liquid.

These simple interpolation formulas are, in fact, what was used by $\mathrm{Ng}$ and Singwi in their quantitative evaluation of the quasiparticle properties of a three-dimensional electron liquid. $^{21}$

\section{B. Modern interpolation formulas}

As it turns out, all the approximations just described do suffer from a major shortcoming: the large $q$ limit is incorrect for in Eq. (26) $\lim _{q \rightarrow \infty} G_{+}(q)=1$ and $\lim _{q \rightarrow \infty} G_{-}(q)=0$, while the correct limits, for which the exact expression is known, is in both cases given by $\lim _{q \rightarrow \infty} G_{ \pm}(q) \propto q^{2}$. 1,42,43

More precisely, the large momentum limit of $G_{+}(q, 0)$ in three dimensions has been obtained by Niklasson, ${ }^{42}$ and Holas $^{43}$ and can be expressed as ${ }^{1}$

$$
\begin{aligned}
G_{+}(q, 0) \simeq & -\frac{\pi}{2 e^{2} k_{F}} \frac{d\left(r_{s} \epsilon_{c}\right)}{d r_{s}} \frac{q^{2}}{k_{F}^{2}}+\frac{2}{3}[1-g(0)] \\
& +\frac{\pi}{3 r_{s} k_{F}^{4}}\left(\frac{9 \pi}{4}\right)^{1 / 3}\left(9\left\langle(\vec{k} \cdot \hat{q})^{4}\right\rangle-9\left\langle(\vec{k} \cdot \hat{q})^{4}\right\rangle_{0}\right. \\
& \left.+\left\langle k^{2}\right\rangle^{2}-\left\langle k^{2}\right\rangle_{0}^{2}\right)
\end{aligned}
$$

In this expression, $\epsilon_{c}$ is the correlation energy per particle, while $g(0)$ is the value of the pair distribution function at the origin. Here, the symbol $\langle\cdots\rangle$ indicates an average over the exact single particle occupation numbers, while $\langle\cdots\rangle_{0}$ represents the corresponding average for noninteracting electrons.

A similar expression holds for the antisymmetric local field $G_{-}(q, 0) .{ }^{1,18,43}$ Using the same notation, we have

$$
\begin{aligned}
G_{-}(q, 0) \simeq & -\frac{\pi}{2 e^{2} k_{F}} \frac{d\left(r_{s} \epsilon_{c}\right)}{d r_{s}} \frac{q^{2}}{k_{F}^{2}}+\frac{1}{3}[4 g(0)-1] \\
& +\frac{\pi}{3 r_{s} k_{F}^{4}}\left(\frac{9 \pi}{4}\right)^{1 / 3}\left(9\left\langle(\vec{k} \cdot \hat{q})^{4}\right\rangle-9\left\langle(\vec{k} \cdot \hat{q})^{4}\right\rangle_{0}\right. \\
& \left.+\left\langle k^{2}\right\rangle^{2}-\left\langle k^{2}\right\rangle_{0}^{2}\right)
\end{aligned}
$$

As a consequence, a new set of suitable formulas must be devised since, as we will show, the large wave-vector limit of the many-body local field factors is quite relevant in the determination of the physical properties of the system.

Diffusion Quantum Monte Carlo (QMC) methods allow the quantitative determination of some of the response functions of an electron liquid as a function of the wave vector. ${ }^{6,7,27}$ While in two dimensions QMC results for both $G_{-}(q)$ and $G_{+}(q)$ are available, only the symmetric local field has been accurately calculated to date in the threedimensional case. Accordingly, we will separate the discussion of the two many-body local fields.

\section{Spin-symmetric many-body field factor $G_{+}$}

Accurate empirical formulas for the static many-body local field $G_{+}(q)$ can be obtained by simply interpolating the results of the available QMC calculations for the threedimensional electron gas. In particular, an analytic fit to such data has been proposed in Ref. 7 and is given by

$$
G_{+}(q)=\frac{C\left(r_{s}\right) q^{2}}{k_{F}^{2}}+\frac{B\left(r_{s}\right) q^{2}}{\gamma\left(r_{S}\right) k_{F}^{2}+q^{2}}+\frac{\alpha\left(r_{s}\right) q^{4}}{k_{F}^{4}} \exp \left[-\frac{\beta\left(r_{s}\right) q^{2}}{k_{F}^{2}}\right] \text {. }
$$

The explicit expressions of the functions $C, B, \alpha, \beta$, and $\gamma$ appearing in Eq. (31) can be found in the original paper. Typical curves obtained with this formula are plotted in Fig. 1 for different electron densities. This analytic fit satisfies the known asymptotic limits both at small and large wave vectors. Since this is the best available formula for $G_{+}(q)$, we will heretofore employ it in all our calculations. 


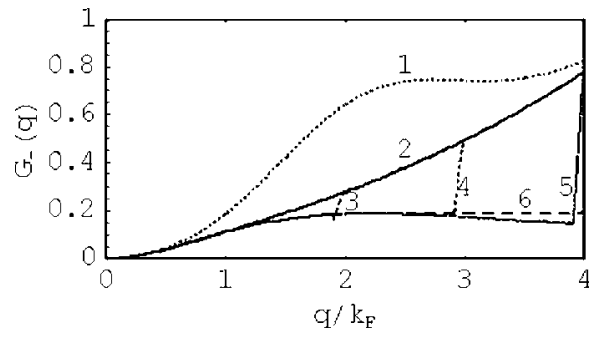

FIG. 2. Alternative approximate expressions for $G_{-}(q, 0)$ in three dimensions. Labels $1-6$ correspond in the order to $G_{-}^{(3)}(q)$, $G_{-}(q), G_{-}^{(2)}(q ; 2), G_{-}^{(2)}(q ; 3), G_{-}^{(2)}(q ; 4)$, and $G_{-}^{(1)}(q)$. Here, $r_{s}=2.0$.

\section{Spin-antisymmetric many-body field factor $G_{-}$}

At variance with the case of a two-dimensional electron liquid, to date, there have been no QMC simulation results reported that could lead to a determination of $G_{-}(q, 0)$ in three dimensions. Accordingly, in order to make progress, we have implemented a number of empirical parametrized formulas designed to reflect all the known exact limiting behaviors as well as any of the relevant features observed in the corresponding quantity in two dimensions.

Proceeding from the simplest to the most complex, we have used in our calculations the following expressions for $G_{-}(q)$.

The simplest consists in a generalization of the Iwamoto and Pines formula to allow for a nonzero, yet finite value for $G_{-}(q)$ at large $q$, i.e.,

$$
G_{-}^{(1)}(q)= \begin{cases}\frac{1}{2}\left(\frac{q^{2}}{q^{2}+q_{\uparrow \uparrow}^{2}}-\frac{q^{2}}{q^{2}+q_{\uparrow \downarrow}^{2}}\right), & q<\sqrt{q_{\uparrow \uparrow} q_{\uparrow \downarrow}} \\ \frac{1}{2} \frac{q_{\uparrow \uparrow}-q_{\uparrow \downarrow}}{q_{\uparrow \uparrow}+q_{\uparrow \downarrow}}, & q>\sqrt{q_{\uparrow \uparrow} q_{\uparrow \downarrow}},\end{cases}
$$

where the notation is the same as in Eq. (26). Clearly, this expression does not still satisfy the large momentum limit, but it will turn useful to test the effect of the large $q$ tail in $G_{-}(q)$ on the actual physical results. The interpolation of Eq. (32) corresponds to the line labeled 6 in Fig. 2.

A possible formula that can be made to satisfy both known $q$ limits is, on the other hand, provided by

$$
G_{-}(q)=\frac{C\left(r_{s}\right) q^{2}}{k_{F}^{2}}+\frac{B_{1}\left(r_{s}\right) q^{2}}{\gamma_{1}\left(r_{s}\right) k_{F}^{2}+q^{2}} .
$$

In this expression, while $C\left(r_{s}\right)$ is the same as in Eq. (31), the function $B_{1}\left(r_{s}\right)$ can be determined from Eqs. (31) and (30) to be given by

$$
B_{1}\left(r_{s}\right)=B\left(r_{s}\right)-1+2 g(0) .
$$

In order to make progress in the calculation [Eq. (34)], a practical, elegant, and simple approximation for $g(0),{ }^{44}$ which we have used in our numerical analysis, is that proposed by Overhauser, ${ }^{18}$

$$
g(0)=\frac{32}{\left(8+3 r_{s}\right)^{2}}
$$

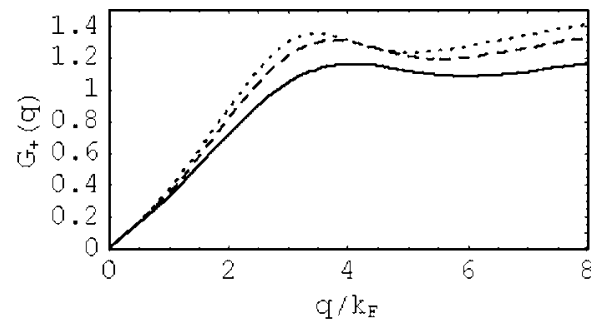

FIG. 3. Wave-vector dependence of the two-dimensional $G_{+}(q, 0)$ as obtained from quantum Monte Carlo simulations. Solid line: $r_{s}=1$; dashed line: $r_{s}=2$; dotted line: $r_{s}=4$ (Ref. 25).

In Eq. (33), the parameter $\gamma_{1}$ is determined by the small momentum limit. Using the relation between the spin susceptibility and the long wavelength limit of $G_{-}(q, 0)$ of Eq. (28), we arrive to the following formula:

$$
\gamma_{1}=\frac{B_{1}\left(r_{s}\right)}{\frac{k_{F}^{2}}{\kappa_{3}^{2}}\left(1+\frac{1}{\mu_{B}^{2}} \frac{\chi_{P}}{\chi_{S_{z} S_{z}}}\right)-C\left(r_{s}\right)},
$$

where now the remaining piece of the puzzle, the spin susceptibility enhancement, can be evaluated from the exact polarization dependence of the correlation energy per electron $\epsilon_{c}\left(r_{s}, \zeta\right)$ via the formula

$$
-\frac{1}{\mu_{B}^{2}} \frac{\chi_{P}}{\chi_{S_{z} S_{z}}}=1-\left(\frac{4}{9 \pi}\right)^{1 / 3} \frac{r_{s}}{\pi}+3\left(\frac{4}{9 \pi}\right)^{2 / 3} r_{s}^{2}\left[\frac{\partial^{2} \epsilon_{c}\left(r_{s}, \zeta\right)}{\partial \zeta^{2}}\right]_{\zeta=0}
$$

where $\zeta$ is the fractional spin polarization.

Values of the correlation energy $\epsilon_{c}$ were obtained from QMC calculations ${ }^{6,45}$ for both the cases of a nonpolarized and a fully polarized three-dimensional electron gas. Since there are no QMC results for the spin stiffness [the second derivative of $\epsilon_{c}$ appearing in Eq. (37)] in three dimensions, an approximate interpolation, proposed by Vosko, ${ }^{45}$ can instead be used,

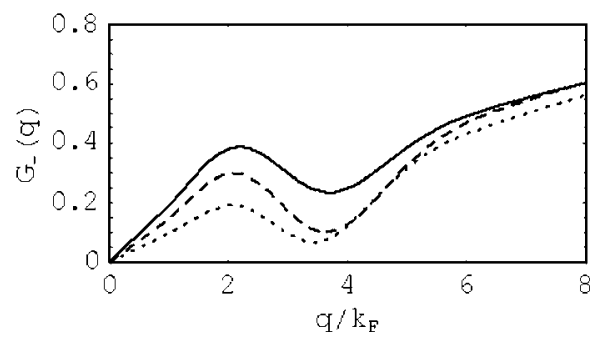

FIG. 4. Wave-vector dependence of $G_{-}(q, 0)$ for a twodimensional electron liquid as obtained from quantum Monte Carlo simulations. Solid line: $r_{s}=1$; dashed line: $r_{s}=2$; dotted line; $r_{s}=4$ (Ref. 26). 


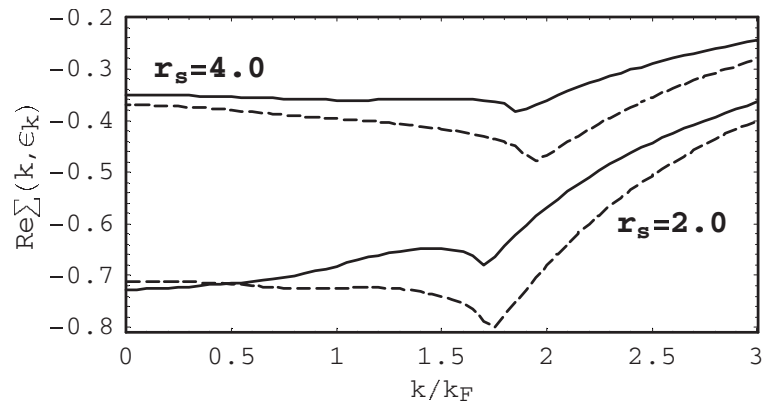

FIG. 5. Real part of the self-energy. Solid line: full theory; dashed line: RPA.

$\left[\frac{\partial^{2} \epsilon_{c}\left(r_{s}, \zeta\right)}{\partial \zeta^{2}}\right]_{\zeta=0} \simeq \frac{\epsilon_{c}\left(r_{s}, 1\right)-\epsilon_{c}\left(r_{s}, 0\right)}{\epsilon_{c}^{R P A}\left(r_{s}, 1\right)-\epsilon_{c}^{R P A}\left(r_{s}, 0\right)}\left[\frac{\partial^{2} \epsilon_{c}^{R P A}\left(r_{s}, \zeta\right)}{\partial \zeta^{2}}\right]_{\zeta=0}$.

This formula makes use of several properties of a threedimensional electron liquid that can be found in the work of Perdew and Wang. ${ }^{46}$ The interpolation of Eq. (33) corresponds to the line labeled 2 in Fig. 2.

One must realize that Eq. (33) has been devised without any input regarding the intermediate $q$ behavior of the function. We have therefore endeavored to construct variations of this formula designed to explore the relevance and the effects of the actual shape of $G_{-}(q)$ on the physical results.

One of the possible alternative variations of Eq. (33) is provided by

$$
G_{-}^{(2)}(q ; a)= \begin{cases}\frac{1}{2}\left(\frac{q^{2}}{q^{2}+q_{\uparrow \uparrow}^{2}}-\frac{q^{2}}{q^{2}+q_{\uparrow \downarrow}^{2}}\right), & q<a k_{F} \\ C\left(r_{s}\right) q^{2}+\frac{B_{1}\left(r_{s}\right) q^{2}}{\gamma_{1}\left(r_{s}\right)+q^{2}}, & q>a k_{F},\end{cases}
$$

which is a simple generalization of the Iwamoto and Pines formula designed to allow an estimate of the effects of the intermediate $q$ regime on the physical properties. This expression corresponds to lines 3, 4, and 5 in Fig. 2.

Finally, an alternative interesting interpolation formula was inspired by the $q$ dependence of the two-dimensional many-body field factors that we have reproduced in Figs. 3 and 4. In these plots, it is clear that, at least in two dimensions, both $G_{-}(q)$ and $G_{+}(q)$ display a peak in the intermediate $q$ region. In order to explore the effect of the existence of such a feature, we have devised the following expression:

$$
G_{-}^{(3)}(q)=C\left(r_{s}\right) \frac{q^{2}}{k_{F}^{2}}+\frac{B_{1}\left(r_{s}\right) q^{2}}{\gamma_{1}\left(r_{s}\right) k_{F}^{2}+q^{2}}+\frac{\alpha\left(r_{s}\right) q^{4}}{k_{F}^{4}} \exp \left(-\beta \frac{q^{2}}{k_{F}^{2}}\right),
$$

where $\alpha$ and $\beta$ are the same quantities appearing in Eq. (31). This last interpolation formula corresponds to line 1 in Fig. 2.

\section{CALCULATING THE ELECTRONIC SELF-ENERGY}

The screened-exchange part of the electron self-energy, as given by Eqs. (8) and (24), does not present any remarkable numerical challenges. On the other hand, a number of comments are in order to describe our handling of the numerical analysis of Eqs. (9) and (25) corresponding to the Coulombhole contributions.

\section{A. Coulomb-hole part of the self-energy}

Two problems require special attention in the evaluation of the Coulomb-hole part of the self-energy.

\section{Handling the plasma pole}

To start with, we notice that the denominator of the density response equation [Eq. (10)] vanishes when

$$
1-\left[1-G_{+}(\vec{q}, \omega)\right] v_{\vec{q}} \operatorname{Re} \chi_{0}(\vec{q}, \omega)=0,
$$

and concomitantly the imaginary part of the Lindhard function vanishes. This singularity is associated with the presence in the spectrum of collective modes (in this case plasmons) of which the dispersion law is represented by the solution $\Omega_{p l}(\vec{q})$ of Eq. (41). The plasmon is Landau damped when it enters the electron-hole continuum. No corresponding singularity occurs for the spin response, for which the correspondent collective excitations (paramagnons) are damped for all momenta.

The real part of the Coulomb-hole self-energy in Eq. (9) can be separated into a plasmonic part and an electron-hole part,

$$
\operatorname{Re} \Sigma_{C H}(\vec{k}, \omega)=\operatorname{Re} \Sigma_{C H}^{(e h)}(\vec{k}, \omega)+\operatorname{Re} \Sigma_{C H}^{(p l)}(\vec{k}, \omega) .
$$

The plasmon contribution to the imaginary part of the dielectric constant can be written as ${ }^{1}$

$$
\left.\operatorname{Im} \frac{1}{\varepsilon(\vec{q}, \omega)}\right|_{\text {plasmon }}=-\pi \frac{\delta\left[\omega-\Omega_{p l}(\vec{q})\right]}{\left|\frac{\partial \operatorname{Re} \varepsilon(\vec{q}, \omega)}{\partial \omega}\right|},
$$

where

$$
\varepsilon(\vec{q}, \omega)=1-\frac{v_{\vec{q}} \chi_{0}(\vec{q}, \omega)}{1+G_{+}(\vec{q}, \omega) v_{\vec{q}} \chi_{0}(\vec{q}, \omega)} .
$$

Approximating $G_{+}(\vec{q}, \omega)$ with its static value, the plasmonic part of Eq. (9) becomes

$$
\begin{aligned}
\operatorname{Re} \Sigma_{c h}^{(p l)}(\vec{k}, \omega)= & -\frac{\hbar k}{m} \int_{0}^{q_{p}\left(r_{s}\right)} \frac{q^{3} d q}{(2 \pi)^{2}} \\
& \times \ln \left|\frac{\Omega_{p l}(q)+\frac{\hbar\left(k^{2}+q^{2}\right)}{2 m}+\frac{\hbar(k q)}{m}-\omega}{\Omega_{p l}(q)+\frac{\hbar\left(k^{2}+q^{2}\right)}{2 m}+\frac{\hbar(k q)}{m}+\omega}\right| \\
& \times\left|\frac{\partial \operatorname{Re} \chi_{0}(q, \omega)}{\partial \omega}\right|_{\omega=\Omega_{p l}(q)}^{-1},
\end{aligned}
$$


where $q_{p}\left(r_{s}\right)$ is the value of the momentum marking the onset of Landau damping for the plasma excitation, i.e., the threshold value of $q$ above which the plasmon branch enters the electron-hole continuum.
As for the electron-hole part, we notice that the integration over frequencies is done in the interval for which $\operatorname{Im} \chi_{0}(q, \omega)$ is finite. We have

$$
\Sigma_{c h}^{(e h)}(\vec{k}, \omega)=-\int \frac{d^{3} q}{(2 \pi)^{3}} \mathcal{P} \int_{\Omega_{l}(q)}^{\Omega_{u}(q)} \frac{d \omega^{\prime}}{\pi} \frac{\operatorname{Im}\left\{v_{\vec{q}}^{2}\left|1-G_{+}\left(\vec{q}, \omega^{\prime}\right)\right|^{2} \chi_{n n}\left(\vec{q}, \omega^{\prime}\right)+3 v_{\vec{q}}^{2}\left|G_{-}\left(\vec{q}, \omega^{\prime}\right)\right|^{2} \chi_{S_{z} S_{z}}\left(\vec{q}, \omega^{\prime}\right)\right\}}{\omega-\omega^{\prime}-\frac{\epsilon_{\vec{k}-\vec{q}}}{\hbar}},
$$

where $\Omega_{u}=\frac{\hbar\left(q^{2}+2 k_{F} q\right)}{2 m}$ and $\Omega_{l}=\frac{\hbar\left(q^{2}-2 k_{F} q\right)}{2 m}$ are the lower and upper limits of the electron-hole continuum for momentum $q$.

\section{Regularizing the ultraviolet catastrophe of the Coulomb-hole part of the self-energy}

There is one more problem associated with the calculation of the real part of the Coulomb-hole self-energy. As a direct inspection will reveal, in view of the large $q$ behavior of the many-body local field factors, the integrand of Eq. (46) remains constant as $q \rightarrow \infty$, leading to an ultraviolet catastrophe. This problem does not arise with the interpolation formulas of the Hubbard type since, by construction, they display an incorrect asymptotic behavior (a constant for large wave vectors) for $G_{ \pm}(q)$. The same problem plagues the corresponding theory of the two-dimensional electron liquid. ${ }^{24}$

Postponing a more detailed discussion of this problem to Sec. VII, it will suffice here to state that we will surmount this obstacle by limiting our analysis to the changes of the self-energy from its value at the Fermi energy. In practice, one defines the function $I(q, k, \omega)$ via the relation

$$
\Sigma_{C H}^{(e h)}(\vec{k}, \omega)=\int \frac{d q}{4 \pi^{3}} I(q, k, \omega),
$$

with

$$
I(q, k, \omega) \simeq e^{2} \kappa_{3}^{2} k_{F}^{2} C^{2} \frac{2}{\pi}\left(\frac{2}{3}+\frac{2 k_{F}^{2}}{15 q^{2}}+\frac{2 \omega m}{\hbar q^{2}}-\frac{k^{2}}{9 q^{2}}\right) .
$$

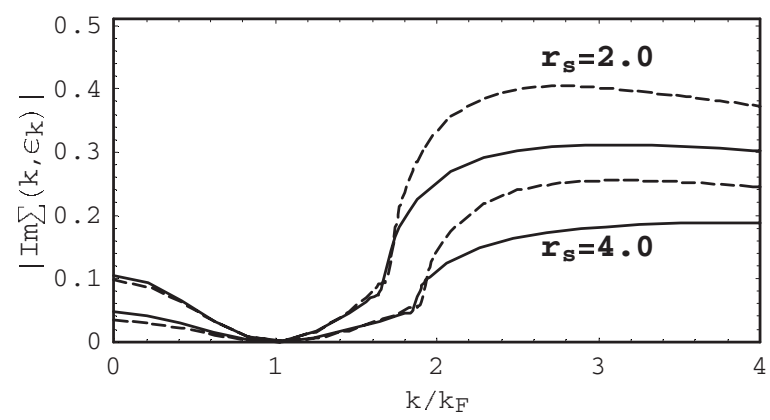

FIG. 6. Imaginary part of the self-energy. Solid line: full theory; dashed line: RPA.
At this point, the self-energy can be defined as the sum of the actual chemical potential $\mu\left(r_{s}\right)$ augmented by an integral of the difference of the original integrand and the value of the latter at the chemical potential. Since when taking the difference the offending behavior cancels out, the final expression is well behaved and can then be computed,

$$
\begin{aligned}
\Sigma(\vec{k}, \omega)= & \mu\left(r_{s}\right)+\int \frac{d^{3} q}{4 \pi^{3}}\left\{I(\vec{k}, \omega, \vec{q})-I\left[\hat{k} k_{F}, \frac{\mu\left(r_{s}\right)}{\hbar}, \vec{q}\right]\right\} \\
& +\Sigma_{S X}(\vec{k}, \omega)-\Sigma_{S X}\left[\hat{k} k_{F}, \frac{\mu\left(r_{s}\right)}{\hbar}\right]+\Sigma_{C H}^{(p l)}(\vec{k}, \omega) \\
& -\Sigma_{C H}^{(p l)}\left[\hat{k} k_{F}, \frac{\mu\left(r_{s}\right)}{\hbar}\right] .
\end{aligned}
$$

If the absolute value of the self-energy is desired, $\Sigma\left(k_{F}, \omega_{F}\right)$ must be gotten by independent means. A possible way is to obtain it from the exact formula

$$
\mu\left(r_{s}\right)=\frac{e^{2}}{2 a_{B} r_{s}^{2}}\left(\frac{9 \pi}{4}\right)^{2 / 3}-\frac{e^{2}}{\pi a_{B} r_{s}}\left(\frac{9 \pi}{4}\right)^{1 / 3}+\varepsilon_{c}-\frac{r_{s}}{3} \frac{d \varepsilon_{c}}{d r_{s}},
$$

where $\varepsilon_{c}$ can be again taken from QMC simulations., ${ }^{1,6,46}$

\section{B. Self-energy: Numerical results}

Representative results for the real and imaginary parts of the on-shell (i.e., for $\omega=\epsilon_{\vec{k}, \sigma}$ ) self-energy $\Sigma[k, \varepsilon(k)]$ of a three-dimensional electron liquid are presented in Figs. (5) and (6) as a function of the wave vector for two values of the density. In these figures, the solid lines represent the fully interacting result obtained using $G_{+}(q, 0)$ and $G_{-}(q, 0)$, respectively, given by Eqs. (31) and (33), while the dashed lines correspond to the RPA calculation obtained by setting $G_{+}(q, 0)=G_{-}(q, 0)=0$. Our RPA results completely agree with the original results of Hedin and Lundqvist. ${ }^{13,47}$

In the real part of the self-energy, the dip associated with the plasmon pole can be easily be observed in Fig. 5. The same can be said for the pronounced corresponding plasmon peak present in the plot of the imaginary part (Fig. 6). Notice that there, the onset of the plasmonic structure is not abrupt. This is in contrast with the situation in the two-dimensional case where the onset is sudden..$^{24,48,49}$ As expected, the value 


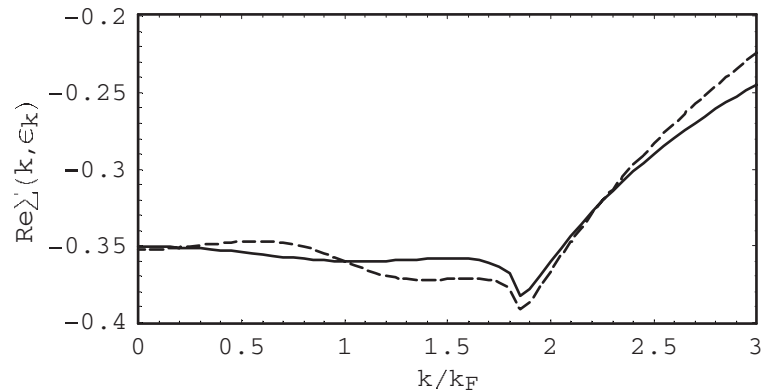

FIG. 7. Comparison between the results obtained for the real part of the self-energy by making use of Eq. (31) for $G_{+}(q, 0)$ and two alternative formulas for $G_{-}(q, 0)$. Solid line: $G_{-}(q, 0)=G_{-}(q)$; dashed line: $G_{-}(q, 0)=G_{-}^{(1)}(q)$. Here, $r_{s}=4.0$.

of $\operatorname{Im} \Sigma[k, \varepsilon(k)]$ vanishes at the Fermi surface, leading to an infinite lifetime for the quasiparticles.

The expression for $G_{+}(q, 0)$ having been set by Eq. (31), the effect of the particular shape of $G_{-}(q, 0)$ on the selfenergy has been examined. A representative plot is provided in Fig. 7 for the real part of $\operatorname{Re} \Sigma[k, \varepsilon(k)]$. An analysis of our results leads us to conclude that, while there appears to be no substantial differences in the general shape of the self-energy when different expressions are used for the spinantisymmetric many-body local field factor, the behavior of the derivatives is altogether a different story.

\section{QUASIPARTICLE EFFECTIVE MASS AND RENORMALIZATION CONSTANT}

The results of our calculations of the quasiparticle effective mass in various approximations are summarized in Table I as well as displayed in Fig. 8. There, one can compare results obtained within a variety of approximations: RPA, $G_{+}$ only, and full theory with both $G_{+}$and $G_{-}$included. One notices that the effect of the density fluctuations is to decrease the effective mass from its RPA value, while the spin fluctuations tend to increase it, the effect being larger for large $r_{s}$ (smaller densities). The same effect has been predicted by Overhauser, ${ }^{50}$ Zhu and Overhauser, ${ }^{18}$ and $\mathrm{Ng}$ and Singwi. ${ }^{21}$

We have also investigated how the use of the full Dyson equation, i.e., Eq. (2), compares with simpler procedure of calculating the quasiparticle energy by employing the OSA of Eq. (5). Our results clearly show that the OSA mass tends to be larger than that obtained through the Dyson equation

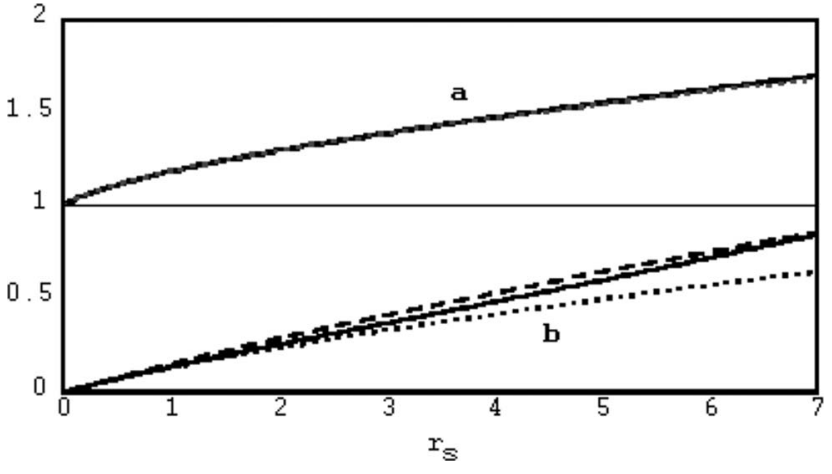

FIG. 8. Effective mass. (a) RPA, (b) using both $G_{+}(q, 0)$ and $G_{-}(q, 0)$, and (c) using $G_{+}(q, 0)$ only. Dashed lines: on-shell approximation; solid lines: Dyson equation.

for large $r_{s}$. More importantly, as one can verify by inspecting Fig. 9, contrary to the two-dimensional case, there is no singularity for the OSA mass in the range of physically accessible densities. Figure 9 displays the key ingredients: the wave-vector and frequency derivatives of the self-energy. With reference to Eq. (5), one must realize that since $\left.\frac{\partial \operatorname{Re} \sum(k, \omega)}{\partial \omega}\right|_{k=k_{F}}$, $\omega=\epsilon_{F} / \hbar$ is in this regime negative, where the two curves in Fig. 9 intersect (as it is the case in two dimensions), a physically spurious divergence of the effective mass would result.

We have also studied the effect of modifying the $q$ dependence of $G_{-}(q, 0)$ by making use of the interpolation formulas introduced in Sec. III. As one can see from Figs. 10-12, making use of $G_{-}^{(2)}(q)$ and $G_{-}^{(2)}(q ; a)$ does not change the effective mass significantly.

We turn next our attention to the effects of including of a peak in the shape of $G_{-}(q, 0)$. As it can be surmised from our results, it is quite clear that the presence of the peak in the expression of $G_{-}$does indeed lead to a significant increase in the effective mass. This is particularly so at larger $r_{s}$ (lower densities). It is clear from Figs. 10-12 that, at least in this regime, a more detailed knowledge of the behavior at intermediate momentum of the spin-antisymmetric many-body local field factor is necessary in order to obtain quantitatively accurate results.

We conclude this section with a discussion of the renormalization constant $Z$ which we have obtained from Eq. (1). The results of our calculations are presented in Fig. 13.

As one can see, charge and spin fluctuations have opposite effects on the renormalization constant. While the former

TABLE I. Effective mass enhancement $m^{*} / m$.

\begin{tabular}{lcccccc}
\hline \hline$r_{s}$ & 1 & 2 & 3 & 4 & 5 & 6 \\
\hline$m_{O S A}^{*}(\mathrm{RPA})$ & 0.969 & 0.995 & 1.030 & 1.068 & 1.109 & 1.153 \\
$m_{D}^{*}(\mathrm{RPA})$ & 0.970 & 0.992 & 1.016 & 1.039 & 1.059 & 1.078 \\
$m_{O S A}^{*}\left(G_{+}\right)$ & 0.945 & 0.944 & 0.947 & 0.953 & 0.958 & 0.965 \\
$m_{D}^{*}\left(G_{+}\right)$ & 0.952 & 0.951 & 0.956 & 0.962 & 0.968 & 0.973 \\
$m_{O S A}^{*}\left(G_{+} \& G_{-}\right)$ & 0.953 & 0.962 & 0.983 & 1.014 & 1.056 & 1.112 \\
$m_{D}^{*}\left(G_{+} \& G_{-}\right)$ & 0.957 & 0.966 & 0.983 & 1.005 & 1.028 & 1.055 \\
\hline \hline
\end{tabular}




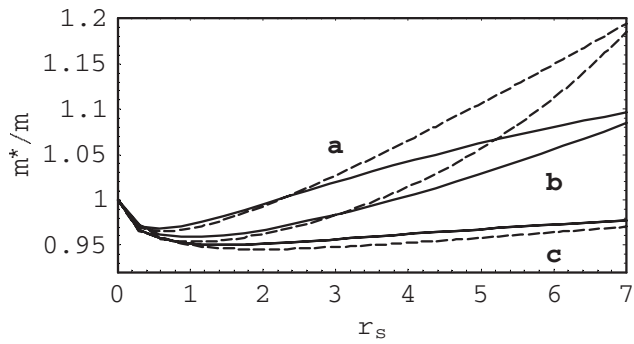

FIG. 9. Quantities entering the effective mass formulas. Group labeled (a): $1+\left.\frac{m}{\hbar^{2} k_{F}} \quad \frac{\partial \operatorname{Re} \Sigma(k, \omega)}{\partial \omega}\right|_{k=k_{F}, \omega=\epsilon_{F} / \hbar}$. Group labeled (b): $\frac{1}{\hbar}\left|\frac{\partial \operatorname{Re} \Sigma(k, \omega)}{\partial \omega}\right|_{k=k_{F}}, \omega=\epsilon_{F} / \hbar$. Dashed lines: RPA; dotted line: $G_{+}$only; solid lines: $G_{+}$and $G_{-}$.

tend to quench it, the latter tend to enhance it raising it to values that actually exceed those obtained in the RPA. A similar result has been obtained by Holm and von Barth using a self-consistent GW approximation. ${ }^{51}$ A discussion of this result is provided in Sec. VII.

\section{LANDAU INTERACTION FUNCTION, SPIN SUSCEPTIBILITY, AND COMPRESSIBILITY}

\section{A. Effective Landé $g$ factor and the many-body spin susceptibility enhancement}

Within the Landau theory of the Fermi liquid, the spin susceptibility can be expressed as ${ }^{1,52}$

$$
\frac{\chi_{s}}{\chi_{s}^{0}}=\frac{m^{*}}{m} \frac{1}{1+F_{0}^{a}} \text {. }
$$

The electron-electron interaction enters this expression through both the effective mass and the antisymmetric Landau parameter $F_{0}^{a}$. The latter can be expressed in terms of the Landau interaction function $f_{\sigma, \sigma^{\prime}}$ as follows:

$$
F_{0}^{a}=\frac{N^{*}(0)}{4} \int d \theta \sin \theta\left[f_{\uparrow \uparrow}(\cos \theta)-f_{\uparrow \downarrow}(\cos \theta)\right],
$$

where $N^{*}(0)$ is the renormalized density of states at the Fermi sea (i.e., it involves the effective mass $m^{*}$ ).

Within the same framework, the effective Landé $g$ factor can be expressed as ${ }^{1}$

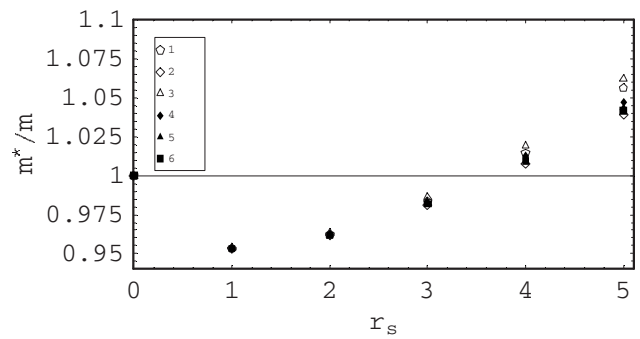

FIG. 10. Effect of the wave-vector dependence of $G_{-}(q, 0)$ on the effective mass (calculated within the on-shell approximation). (1) $G_{-}(q),(2) G_{-}^{(1)}(q),(3) G_{-}^{(2)}(q ; 1),(4) G_{-}^{(2)}(q ; 2),(5) G_{-}^{(2)}(q ; 3)$, and $(6) G_{-}^{(2)}(q ; 4)$.

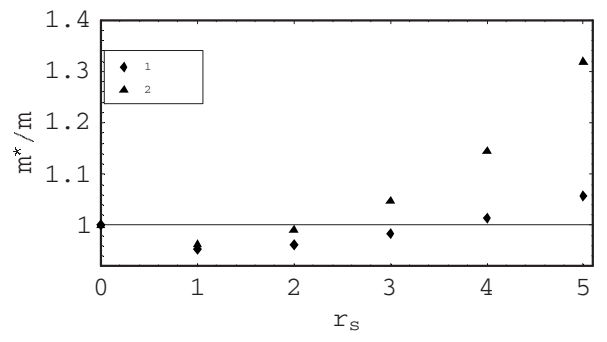

FIG. 11. Effect of the existence of a peak in the wave-vector dependence of $G_{-}(q, 0)$ on the effective mass (calculated within the on-shell approximation). (1) $G_{-}(q)$ and $(2) G_{-}^{(3)}(q)$.

$$
\frac{g^{*}}{g}=\frac{1}{1+F_{0}^{a}} \text {. }
$$

Taking the derivative of the quasiparticle energy with respect to the occupation number, as done in Ref. 22, we can express the interaction function in terms of known quantities (charge and spin response functions and many-body local field factors) as follows:

$$
\begin{aligned}
f_{\uparrow \uparrow}= & F_{\uparrow \uparrow}-\left(v_{q}+v_{q}^{2}\left\{\left[1-G_{+}(q)\right]^{2} \chi_{C}(q, 0)\right.\right. \\
& \left.\left.+\left[G_{-}(q)\right]^{2} \chi_{S_{z} S_{z}}(q, 0)\right\}\right)\left.\right|_{\vec{q}=\vec{k}-\vec{p}}, \\
f_{\uparrow \downarrow}= & F_{\uparrow \downarrow}-\left.2 v_{q}^{2}\left\{\left[G_{-}(q)\right]^{2} \chi_{S_{z} S_{z}}(q, 0)\right\}\right|_{\vec{q}=\vec{k}-\vec{p}},
\end{aligned}
$$

where the quantities $F_{\uparrow \uparrow}$ and $F_{\uparrow \downarrow}$ are here defined as follows:

$$
\begin{aligned}
F^{\uparrow \uparrow}= & -\int \frac{d^{3} q}{(2 \pi)^{3}} \int_{-\infty}^{\infty} \frac{d \varepsilon}{2 \pi} v_{q}^{2}\left(\left\{\left[\frac{1-G_{+}(q)}{\varepsilon_{+}(q, \omega)}\right]^{2}+\left[\frac{G_{-}(q)}{\varepsilon_{-}(q, \omega)}\right]^{2}\right.\right. \\
& \left.-2 \frac{\left[1-G_{+}(q)\right] G_{-}(q)}{\varepsilon_{+}(q, \omega) \varepsilon_{-}(q, \omega)}\right\} \widetilde{G}^{+}+4\left[\frac{G_{-}(q)}{\varepsilon_{-}(q, \omega)}\right]^{2} G_{0}(\vec{k}-\vec{q} \\
& \left.\left.\varepsilon_{\vec{k}}-\varepsilon\right) G_{0}\left(\vec{p}-\vec{q}, \varepsilon_{\vec{p}}-\varepsilon\right)\right)
\end{aligned}
$$

and

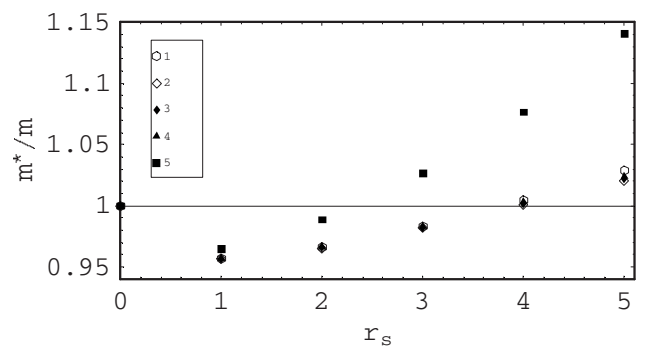

FIG. 12. Effect of the wave-vector dependence of $G_{-}(q, 0)$ on the effective mass (calculated by solving Dyson's equation). (1) $G_{-}(q),(2) G_{-}^{(2)}(q ; 2),(3)-G_{-}^{(2)}(q ; 3),(4)-G_{-}^{(2)}(q ; 4)$, and (5) $G_{-}^{(3)}$ $\times(q)$ 


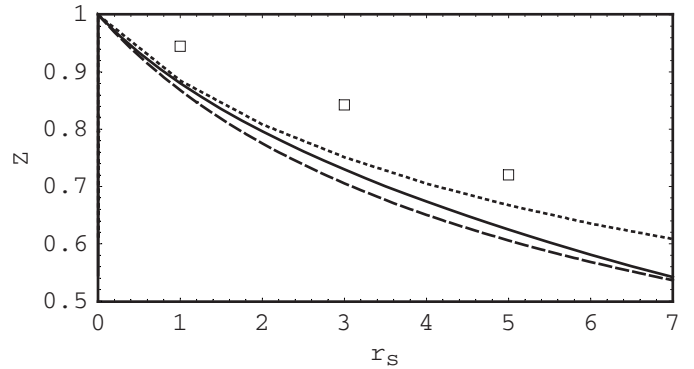

FIG. 13. Quasiparticle renormalization constant $Z$. Solid line: full theory; dashed line: RPA; dotted line: including $G_{+}(q, 0)$ only; squares: QMC results from Ref. 10.

$$
\begin{aligned}
F^{\uparrow \downarrow}= & -\int \frac{d^{3} q}{(2 \pi)^{3}} \int_{-\infty}^{\infty} \frac{d \varepsilon}{2 \pi} v_{q}^{2}\left(\left\{\left[\frac{1-G_{+}(q)}{\varepsilon_{+}(q, \omega)}\right]^{2}+\left[\frac{G_{-}(q)}{\varepsilon_{-}(q, \omega)}\right]^{2}\right.\right. \\
& \left.+2 \frac{\left[1-G_{+}(q)\right] G_{-}(q)}{\varepsilon_{+}(q, \omega) \varepsilon_{-}(q, \omega)}\right\} \widetilde{G^{+}}+4\left[\frac{G_{-}(q)}{\varepsilon_{-}(q, \omega)}\right]^{2} G_{0}(\vec{k}+\vec{q} \\
& \left.\left.\varepsilon_{\vec{k}}+\varepsilon\right) G_{0}\left(\vec{p}-\vec{q}, \varepsilon_{\vec{p}}-\varepsilon\right)\right) .
\end{aligned}
$$

In Eqs. (56) and (57), $G_{0}(\vec{k}, \varepsilon)$ is the noninteracting single particle Green's function and the quantity $\widetilde{G}^{+}$is in turn defined as

$$
\widetilde{G^{+}}=\left[G_{0}\left(\vec{k}+\vec{q}, \varepsilon_{\vec{k}}+\varepsilon\right)+G_{0}\left(\vec{k}-\vec{q}, \varepsilon_{\vec{k}}-\varepsilon\right)\right] G_{0}\left(\vec{p}-\vec{q}, \varepsilon_{\vec{p}}-\varepsilon\right) .
$$

Moreover, the quantities $\varepsilon_{+}(q, \omega)$ and $\varepsilon_{-}(q, \omega)$ are suitably defined dielectric constants, respectively, given by

$$
\varepsilon_{+}(q, \omega)=1-v_{q}\left[1-G_{+}(q, \omega)\right] \chi_{0}(q, \omega)
$$

and

$$
\varepsilon_{-}(q, \omega)=1+v_{q} G_{-}(q, \omega) \chi_{0}(q, \omega),
$$

of which the first one can be recognized as the electron-testcharge dielectric constant. ${ }^{1}$

With the help of these expressions, we have calculated the effective Landé $g$ factor using our interpolation formulas for $G_{+}(q, 0)$ and $G_{-}(q, 0)$ to account for many-body effects beyond RPA. A comparison between the effective Landé factor obtained using the full theory and that obtained using the RPA is presented in Fig. 14. Here, the effective mass used in Eq. (52) was calculated within the OSA.

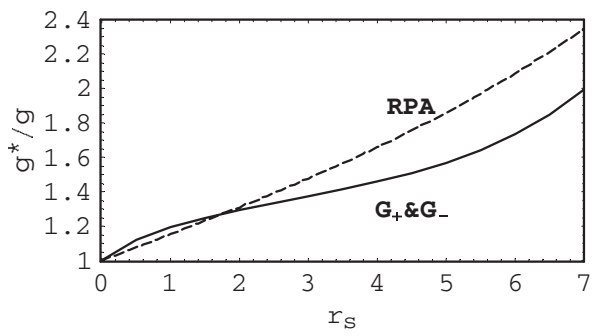

FIG. 14. Effective Landé $g$ factor. Solid line: full theory; dashed line: RPA.

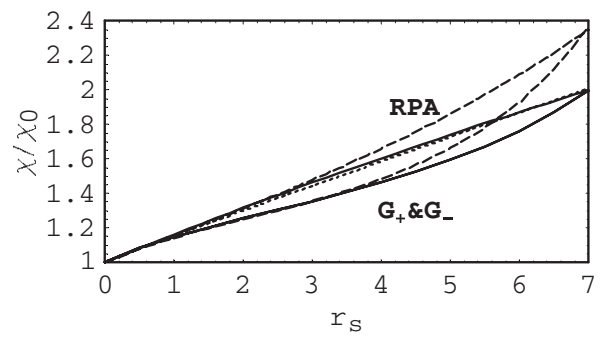

FIG. 15. Spin susceptibility enhancement. Dashed lines: full theory with on-shell approximation effective mass; solid lines: full theory with Dyson effective mass; dotted line: QMC.

The corresponding spin susceptibility enhancement, calculated using Eq. (51), is plotted in Fig. 15. The solid lines are obtained if the Dyson's equation effective mass is used, while the dashed lines represent the result obtained when the OSA mass is used. The dotted line represents the QMC results. ${ }^{46}$ As it can be seen, when using the Dyson effective mass, the RPA is in excellent quantitative agreement with the QMC data.

As done for the effective mass, we have investigated the effect of the presence of a peak in the intermediate wavevector range of $G_{-}(q, 0)$ on the spin susceptibility. A comparison between the spin susceptibility enhancements obtained using our interpolation formulas $G_{-}(q)$ and $G_{-}^{(3)}\left(q k_{F}\right)$ is presented in Fig. 16. Again, a precise knowledge of $G_{-}$is crucial for the accuracy of this type of analysis. As it turns out, the lack of accuracy in $\chi_{s}$ can be traced to the uncertainty in the value of the effective mass. These particular calculations were performed by making use of the OSA mass.

\section{B. Compressibility}

Within the Landau theory of the Fermi liquid, the (proper) compressibility of the electron liquid can be obtained from the general relation ${ }^{1}$

$$
\frac{K}{K_{0}}=\frac{m^{*}}{m} \frac{1}{1+F_{0}^{s}},
$$

where the symmetric Landau parameter $F_{0}^{s}$ can be in turn calculated in exactly the same way as its antisymmetric counterpart.

The corresponding results are presented Fig. 17. It can be noticed that the RPA gives quite accurate results (i.e., results

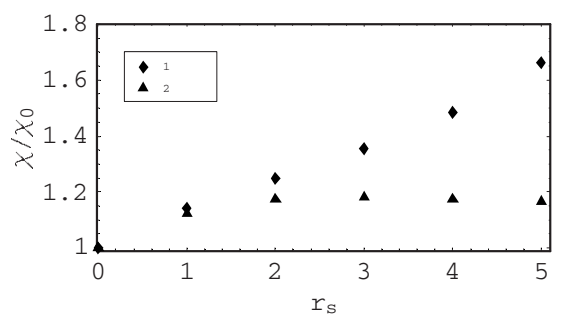

FIG. 16. Effect of the existence of a peak in the wave-vector dependence of $G_{-}(q, 0)$ on the spin susceptibility. Diamonds, 1: $G_{-}(q)$; triangles, $2: G_{-}^{(3)}(q)$. 
in agreement with the available QMC calculations) in the full metallic density range. This remains true also up to relatively low densities. The calculations of this section were all performed using the OSA mass.

\section{DISCUSSION AND CONCLUSIONS}

We have carried out an extensive numerical analysis of the quasiparticle properties of a three-dimensional paramagnetic Fermi liquid within the framework of the many-body local field approach. Within this theory, in the absence of spin polarization, all the many-body effects beyond the RPA can be expressed in terms of the two functions $G_{+}(q, \omega)$ and $G_{-}(q, \omega)$, respectively, accounting for density and spin fluctuations. To date, in view of a lack of knowledge of their frequency dependence, $, 153,54$ it has been a common practice to approximate these quantities with their static limit. The latter is, on the other hand, approximately known only for the two-dimensional case. In three dimensions, only the static spin-symmetric many-body local field factor $G_{+}(q, 0)$ is approximately known. It has therefore proven necessary in this case to devise and explore the effects of a number of model interpolation formulas for the spin-antisymmetric counterpart $G_{-}(q, 0)$.

The bulk of the results of our numerical analysis for the self-energy is represented in Figs. 5-7, where we have chosen to plot the on-shell value of both the real and the imaginary parts of the self-energy as functions of the wave vector. There, a direct comparison is also made between the full theory and the results obtained in RPA.

In the evaluation of the self-energy, we have identified a problem with one of the key quadratures that appears to have escaped notice in the previous work. A direct inspection reveals that in view of the actual large $q$ behavior of the manybody local field factors, the integral of Eq. (46) displays an ultraviolet catastrophe. Although unphysical, this feature of the theory is hardly unexpected since our approximations have all been devised to handle the low energy sector of the excitations of the electron liquid. The impasse can be resolved by noticing that a physically satisfactory (i.e., finite) quantity can be obtained if one abandons the idea of calculating the self-energy and limits oneself to calculate the change in the value of the self-energy as one moves away from the Fermi energy. This amounts to state that the present formulation cannot address the issue of the calculation of the value of the (interacting) chemical potential of the system. It can also be remarked that, quite consistently, this limitation of the theory is equivalent to the loss of knowledge about the self-energy one must endure when employing Eq. (14) for its determination.

By evaluating suitable derivatives of the self-energy, we have been able to extract the Coulomb effective mass and the quasiparticle renormalization constant $Z$. The results for the effective mass are provided in Table I, while in Figs. 8-12, we have displayed how the results of the calculations vary with the way the effective mass is extracted (on-shell vs Dyson's equation methods) and with the specific many-body local field model chosen. Figure 13 contains in turn various curves of $Z$ plotted as a function of density.

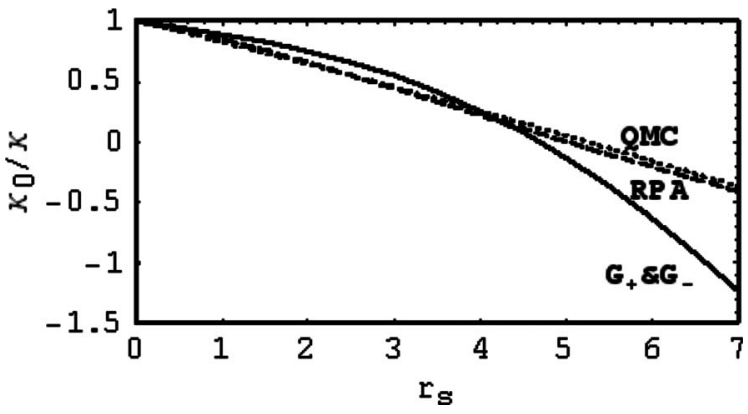

FIG. 17. Compressibility modulus. Solid line: full theory including both $G_{+}(q, 0)$ and $G_{-}(q, 0)$; dashed line: RPA; dotted line: QMC.

We notice first that evaluating the effective mass within the popular on-shell approximation [see Eq. (5)] does tend to lead to values that are higher than those obtained by solving Dyson's equation [see Eq. (3)]. On the other hand, within the density range of interest, the spurious divergence of the onshell effective mass as a function of $r_{s}$ that, as discussed in Ref. 24 plagues the two-dimensional electron liquid case is not observed.

Our results clearly show that the effect of the density fluctuations as accounted through the symmetric many-body local field $G_{+}$is to decrease the effective mass from its RPA value, while the spin fluctuations, accounted via $G_{-}$, tend to increase it. The opposite occurs for the renormalization constant. We find that while charge fluctuations tend to increase $Z$ with respect to its RPA value, the further inclusion of spin fluctuations does quench it (see Fig. 13). The observed trends become more important as $r_{s}$ increases (smaller densities). Notice that all the calculated values of $Z$ are clearly lower than the QMC data reported in Ref. 10. The reason for this discrepancy is currently unknown but one must be wary of the rather approximate way the QMC results for $Z$ were obtained.

The fact that allowing for $G_{+}$leads to a value of $Z$ larger than in RPA can be understood by observing that, as it is clear from its formulation and clearly exemplified in Fig. 6, the RPA tends to overestimate the effects of the plasma mode, thereby resulting in a smaller oscillator strength for the quasiparticles. The further addition of $G_{-}$, by essentially accounting also for spin excitations, tends, on the other hand, to weaken the quasiparticle pole. This behavior is also observed in the two-dimensional electron liquid. ${ }^{24}$ On the other hand, while for the two-dimensional case the full theory results in a value of $Z$ which is lower than the RPA value, in the present case, it leads to a value close albeit larger than the latter. One must, however, keep in mind that while in the two-dimensional case $G_{-}(q, 0)$ is known and does indeed display a characteristic peak (see Fig. 4), the results plotted in Fig. 13 have been obtained by making use of the expression of Eq. (33) which does not.

By design, our calculations have been carried out by employing a number of increasingly complex interpolation formulas for $G_{+}(q, 0)$ and $G_{-}(q, 0)$. This has allowed us to explore which of the quasiparticle properties are reasonably amenable to calculation within the present theoretical frame- 
work. While several features of the local field factors appear to be unimportant, we have been lead to conclude that the current lack of knowledge about the existence of an intermediate peak in the wave-vector dependence of $G_{-}(q, 0)$ severely limits the possibility of carrying out an accurate quantitative evaluation of the effective mass within this formalism. In particular, we have shown that the presence (as compared to a lack) of a peak in the expression of $G_{-}$does lead to a significant increase in the effective mass. Hopefully, future QMC work will come to the rescue in this respect.

Following the procedure employed in Ref. 22 by taking functional derivatives of the quasiparticle energy with respect to the occupation numbers, we have derived a number of useful expressions for the Landau parameters in terms of many-body local fields. This has allowed us to evaluate the anomalous Landé $g$ factor (see Fig. 14), the spin susceptibility (see Figs. 15 and 16), and the proper compressibility of the system (see Fig. 17). It is manifested that in all cases, the effective mass represents the crucial quantity. In particular, this is true for the spin susceptibility $\chi_{s} / \chi_{s}^{0}$ and the compressibility $K / K_{0}$ in view of the their common mass enhancement prefactor. Approximations that lead to a larger effective mass correspond to larger values of $\chi_{s}$ and $K$. One can observe that the RPA results are in good agreement with most of the QMC data over the elemental metallic density range..$^{55}$ This is particularly true for the compressibility.

It should be understood that within the present theory, the calculation of $\chi_{s} / \chi_{s}^{0}$ and $K / K_{0}$ acquires significance only as a check of the self-consistency of the approach. As discussed in Sec. III B, the QMC values of these quantities are, in fact, used as inputs in establishing the interpolation formulas for $G_{+}(q, 0)$ and $G_{-}(q, 0)$ (in particular, their long wavelength limits). It is clear from Figs. 15 and 17 that consistency is reasonable up to $r_{s} \simeq 5$, the many-body enhancement of the spin susceptibility being somewhat less accurate.

We conclude our discussion by remarking that also for the three-dimensional electron liquid, as already observed for the two-dimensional case in Refs. 38 and 56 attempting to go beyond the RPA by including only $G_{+}$leads to inaccurate and, in fact, unphysical results.

\section{ACKNOWLEDGMENTS}

The authors would like to acknowledge useful discussions with A. W. Overhauser and G. Vignale. The work of G.E.S. was partially supported by a grant from the Purdue Research Foundation.

\section{APPENDIX: LINE-RESIDUE DECOMPOSITION}

In this appendix, we present a mathematically equivalent decomposition of the quasiparticle self-energy introduced by Quinn and Ferrell. ${ }^{14,15}$ The integral over frequency defining the self-energy in Eq. (14) can be analytically continued into the lower complex half-plane, resulting in an integration over the imaginary axes and a residue contribution.

Here, $\Sigma(\mathbf{k}, \omega)$ is expressed as the following sum:

$$
\Sigma(\mathbf{k}, \omega)=\Sigma_{X}(\mathbf{k})+\Sigma_{\text {line }}(\mathbf{k}, \omega)+\Sigma_{\text {res }}(\mathbf{k}, \omega),
$$

where we have defined the (real) Hartree-Fock self-energy ${ }^{1}$

$$
\Sigma_{X}(\mathbf{k})=-\frac{2 e^{2} k_{F}}{\pi}\left(\frac{1}{2}+\frac{k_{F}^{2}-k^{2}}{4 k k_{F}} \ln \left|\frac{k_{F}-k}{k_{F}+k}\right|\right),
$$

the line integral (real) contribution

$$
\begin{aligned}
\Sigma_{\text {line }}(\mathbf{k}, \omega)= & -\int \frac{d^{3} q}{(2 \pi)^{3}} v_{\mathbf{q}} \int_{-\infty}^{\infty} \frac{d \omega^{\prime}}{2 \pi}\left[\frac{1}{\varepsilon\left(\mathbf{q}, i \omega^{\prime}\right)}\right. \\
& -1] \frac{1}{\omega+i \omega^{\prime}-\epsilon_{\mathbf{k}-\mathbf{q}} / \hbar},
\end{aligned}
$$

and the "residue" contribution

$$
\begin{aligned}
\Sigma_{r e s}(\mathbf{k}, \omega)= & \int \frac{d^{3} q}{(2 \pi)^{3}} v_{\mathbf{q}}\left[\frac{1}{\varepsilon\left(\mathbf{q}, \omega-\epsilon_{\mathbf{k}-\mathbf{q}} / \hbar\right)}-1\right][\Theta(\omega \\
& \left.\left.-\epsilon_{\mathbf{k}-\mathbf{q}} / \hbar\right)-\Theta\left(-\epsilon_{\mathbf{k}-\mathbf{q}} / \hbar\right)\right] .
\end{aligned}
$$

Within this decomposition, it is the "line" contribution which requires regularization for an ultraviolet divergence. In these expressions, the "dielectric constant" $\varepsilon$ is defined as

$$
\begin{aligned}
\frac{1}{\varepsilon(\mathbf{q}, \omega)}= & 1+v_{\mathbf{q}}\left|1-G_{+}\left(\mathbf{q}, \omega^{\prime}\right)\right|^{2} \chi_{n n}\left(\mathbf{q}, \omega^{\prime}\right) \\
& +3 v_{\mathbf{q}}\left|G_{-}\left(\mathbf{q}, \omega^{\prime}\right)\right|^{2} \chi_{S_{z} S_{z}}\left(\mathbf{q}, \omega^{\prime}\right) .
\end{aligned}
$$

*Present address: Department of Physics and Astronomy, University of Tennessee, Knoxville, TN 37996-1200, USA.

${ }^{1}$ G. F. Giuliani and G. Vignale, Quantum Theory of The Electron Liquid (Cambridge University Press, Cambridge, 2005).

${ }^{2}$ W.-D. Schöne and A. G. Eguiluz, Phys. Rev. Lett. 81, 1662 (1998).

${ }^{3}$ A. Fleszar, R. Stumpf, and A. G. Eguiluz, Phys. Rev. B 55, 2068 (1997).

${ }^{4}$ B. C. Larson, J. Z. Tischler, E. D. Isaacs, P. Zschack, A. Fleszar, and A. G. Eguiluz, Phys. Rev. Lett. 77, 1346 (1996).

${ }^{5}$ Y. Takada, Phys. Rev. Lett. 87, 226402 (2001).

${ }^{6}$ D. M. Ceperley and B. J. Alder, Phys. Rev. Lett. 45, 566 (1980).

${ }^{7}$ S. Moroni, D. M. Ceperley, and G. Senatore, Phys. Rev. Lett. 75,
689 (1995).

${ }^{8}$ S. Moroni, D. M. Ceperley, and G. Senatore, Phys. Rev. Lett. 69, 1837 (1992).

${ }^{9}$ G. Senatore, S. Moroni, and D. M. Ceperley, in Quantum Monte Carlo Methods in Physics and Chemistry, edited by M. P. Nightingale and C. J. Umrigar (Kluwer, Dordrecht, 1999).

${ }^{10}$ G. Ortiz and P. Ballone, Phys. Rev. B 50, 1391 (1994).

${ }^{11}$ J. Hubbard, Proc. R. Soc. London, Ser. A 276, 238 (1963).

${ }^{12}$ T. M. Rice, Ann. Phys. (N.Y.) 31, 100 (1965).

${ }^{13}$ L. Hedin and S. Lundqvist, Solid State Phys. 23, 1 (1969).

${ }^{14}$ J. J. Quinn and R. A. Ferrell, J. Nucl. Energy, Part C 2, 18 (1961).

${ }^{15}$ J. J. Quinn and R. A. Ferrell, Phys. Rev. 112, 812 (1958).

${ }^{16}$ S. Yarlagadda and G. F. Giuliani, Phys. Rev. B 49, 7887 (1994). 
${ }^{17}$ S. Yarlagadda and G. F. Giuliani, Phys. Rev. B 49, 14172 (1994).

${ }^{18}$ X. Zhu and A. W. Overhauser, Phys. Rev. B 33, 925 (1986).

${ }^{19}$ S. Rahman and G. Vignale, Phys. Rev. B 30, 6951 (1984).

${ }^{20}$ T. K. Ng and K. S. Singwi, Phys. Rev. B 34, 7738 (1986).

${ }^{21}$ T. K. Ng and K. S. Singwi, Phys. Rev. B 34, 7743 (1986).

${ }^{22}$ S. Yarlagadda and G. F. Giuliani, Phys. Rev. B 49, 14188 (1994).

${ }^{23}$ S. Yarlagadda and G. F. Giuliani, Solid State Commun. 69, 677 (1989).

${ }^{24}$ R. Asgari, B. Davoudi, M. Polini, G. F. Giuliani, M. P. Tosi, and G. Vignale, Phys. Rev. B 71, 045323 (2005).

${ }^{25}$ B. Davoudi, M. Polini, G. F. Giuliani, and M. P. Tosi, Phys. Rev. B 64, 153101 (2001).

${ }^{26}$ B. Davoudi, M. Polini, G. F. Giuliani, and M. P. Tosi, Phys. Rev. B 64, 233110 (2001).

${ }^{27}$ M. Corradini, R. DelSole, G. Onida, and M. Palummo, Phys. Rev. B 57, 14569 (1998).

${ }^{28}$ C. Attaccalite, S. Moroni, P. Gori-Giorgi, and G. B. Bachelet, Phys. Rev. Lett. 88, 256601 (2002).

${ }^{29}$ M. Crommie, C. Lutz, and D. Eigler, Nature (London) 363, 3384 (1993).

${ }^{30}$ K. Kanisawa, M. J. Butcher, H. Yamaguchi, and Y. Hirayama, Phys. Rev. Lett. 86, 3384 (2001).

${ }^{31}$ B. G. Briner, P. Hofmann, M. Doering, H.-P. Rust, E. W. Plummer, and A. M. Bradshaw, Phys. Rev. B 58, 13931 (1998).

${ }^{32}$ G. F. Giuliani and G. E. Simion, Solid State Commun. 127, 789 (2003).

${ }^{33}$ G. E. Simion and G. F. Giuliani, Phys. Rev. B 72, 045127 (2005).

${ }^{34}$ G. Vignale and K. S. Singwi, Phys. Rev. B 32, 2156 (1985).

${ }^{35}$ A discussion of the physical meaning of "local approximation" can be found in Ref. 1 .

${ }^{36}$ C. A. Kukkonen and A. W. Overhauser, Phys. Rev. B 20, 550 (1979).

${ }^{37}$ D. R. Hamann and A. W. Overhauser, Phys. Rev. 143, 183 (1966).

${ }^{38}$ G. E. Santoro and G. F. Giuliani, Phys. Rev. B 39, 12818 (1989).
${ }^{39}$ We follow here the original terminology. The renormalization aspect of the problem can be carried more into focus by separating the degrees of freedom in terms of "fast" and "slow" electronic states as opposed to "test electrons" and "medium." The procedure is, however, formally equivalent to that given in the text and the results are the same.

${ }^{40}$ N. Iwamoto and D. Pines, Phys. Rev. B 29, 3924 (1984).

${ }^{41}$ D. J. W. Geldart and S. H. Vosko, Can. J. Phys. 44, 2137 (1966).

${ }^{42}$ G. Niklasson, Phys. Rev. B 10, 3052 (1974).

${ }^{43}$ A. Holas, in Strongly Coupled Plasma Physics, edited by F. J. Rogers and H. E. deWitt (Plenum, New York, 1987).

${ }^{44}$ Using an approximative expression for $g(0)$ will introduce small errors comparable to those generated by the unknown behavior of $G_{-}$in the intermediate momentum range.

${ }^{45}$ S. H. Vosko, L. Wilk, and M. Nusair, Can. J. Phys. 58, 1200 (1980).

${ }^{46}$ J. P. Perdew and Y. Wang, Phys. Rev. B 45, 13244 (1992).

${ }^{47}$ L. Hedin, Phys. Rev. 139, A796 (1965).

${ }^{48}$ G. F. Giuliani and J. J. Quinn, Phys. Rev. B 26, 4421 (1982).

${ }^{49}$ P. Hawrylak, G. Eliasson, and J. J. Quinn, Phys. Rev. B 37, 10187 (1988).

${ }^{50}$ A. W. Overhauser, Phys. Rev. B 3, 1888 (1971).

${ }^{51}$ B. Holm and U. von Barth, Phys. Rev. B 57, 2108 (1998).

${ }^{52}$ L. D. Landau, Sov. Phys. JETP 3, 930 (1956).

${ }^{53}$ Z. Qian and G. Vignale, Phys. Rev. B 65, 235121 (2002).

${ }^{54}$ For a discussion of some of the notions regarding the frequency dependence of the many-body local field factors, see Ref. 1.

${ }^{55}$ Interestingly, the RPA value for the density dependent spin susceptibility enhancement has been reproduced in a variety of often allegedly inequivalent ways (see, for instance, the discussion in Ref. 57), the result taking the name of consensus curve. A particularly elegant and compact expression can be found in Ref. 56.

${ }^{56}$ S. Yarlagadda and G. F. Giuliani, Phys. Rev. B 40, 5432 (1989).

${ }^{57}$ T. Kushida, J. C. Murphy, and M. Hanabusa, Phys. Rev. B 13, 5136 (1976). 